\title{
QUEUE DISCHARGE HEADWAY: CHARACTERISTICS, DISTRIBUTIONS AND EFFECTS ON SATURATION FLOW RATES AT SELECTED SIGNALIZED INTERSECTIONS IN LAGOS
}

\author{
Olanrewaju Akinfala ${ }^{1}$, Folorunso Ogunwolu ${ }^{2}$, Emmanuel Ege ${ }^{3}$, \\ Shakirudeen Odunuga ${ }^{4}$ \\ 1,3,4 Department of Geography, University of Lagos, Lagos, Nigeria, \\ ${ }^{2}$ Department of Systems Engineering, University of Lagos, Lagos, Nigeria
}

Received 15 November 2021; accepted 26 December 2021

\begin{abstract}
Saturation flow rate (SFR) and headway are highly localized essential parameters for accurate and effective performance analysis and optimization of signalized intersections. This study examined the saturation flow rate (SFR) and characterized headways at eight signalized intersection approaches, six of which were ideal while two were typical approaches of Lagos conditions. Over 700 cycle to cycle headway data was collected and analyses such as Fuzzy level 1; statistical tests such as Wilcoxon-Signed rank, Mann-Whitney and ShapiroWilks tests were performed on the observed headways. The average SFR observed for ideal approaches was $1,927 \mathrm{veh} / \mathrm{hr} /$ lane which is higher than values observed in many developed countries, while at typical approaches, it was $1,342 \mathrm{veh} / \mathrm{hr} / \mathrm{lane}$. Statistically, SFR differed significantly during peak and off-peak periods (230 veh/hr/lane) and at ideal and typical approaches (between 460-750 veh/hr/lane). Across all traffic conditions observed, the Burr group of distributions were the overall best fit for modelling headways. A fuzzy-based SFR of $1,789 \mathrm{veh} / \mathrm{hr} /$ lane was proposed. This study shows that indeed, headway and SFR differ considerably spatiotemporally. Therefore, appropriate SFR and headway values which reflect actual operating conditions must be adopted to ensure optimal operational performance at signalized intersections.
\end{abstract}

Keywords: saturation flow rates, burr distribution, headways, locally calibrated data, performance analysis.

\section{Introduction}

Signalized intersections are critical points in any road network, particularly in urban areas. They are also a source of conflict and congestion in urban areas. They are thus important points of interest if the urban traffic system is to be utilized more effectively (Stokes, 1988). Consequently, optimizing their operations is essential towards increasing network performance in urban areas. Traffic signals are primarily installed to ensure there is safe and efficient allocation of right-of-way among conflicting road users (Koonce et al., 2008). The latter objective cannot be achieved without sound understanding and accurate quantification of saturation flow rate, as it is used to calculate start-up lost time and end-gain times which are parameters for the determination of

${ }^{2}$ Corresponding author: fogunwolu@unilag.edu.ng 
effective green times. Effective green times and saturation flow rate are the key parameters used for capacity calculation and for the development of many signalized intersection performance models that are based on delay and queue length (Akcelik, 1998). The use of inaccurate saturation flow rate data to develop timing plans results in congestion, more intense traffic delays, increased fuel consumption and pollution and a higher frequency of collisions (Fornalchyk, Mohyhla and Hilevych, 2013). Conceptually, saturation flow rate is the maximum constant departure rate from the queue during the green period, remaining largely constant throughout the rest of the green or until the queue is completely served or exhausted (Agent and Crabtree, 1982). It is a key traffic intersection performance assessment or prediction parameter and one of the two parameters used to quantify intersection capacity as shown in (Eq. 1).

$c=s \times \frac{G}{C_{y}}$

where $c$ is capacity, $s$ is saturation flow rate and $G / C y$ is effective green to cycle time ratio. The value of $c$ calculated above is then used to compute volume to capacity ratio as shown in (Eq. 2).

$X_{i}=\frac{v_{i}}{c_{i}}$

where $X i$ is saturation ratio of a particular movement group $i, v_{i}$ is demand or predicted volume for movement $i$ and $c_{i}$ is the capacity for movement $\mathrm{i}$. The value of $\mathrm{Xi}$ above forms the basis for the development of many performance models such as those for delay and queue length.

(HCM, 2010) defined the SFR as the maximum flow at a traffic lane, and it is typically measured at the stop-line during the green interval at the approach of a signalized intersection. It has an inverse relationship with saturation headway (Ranasinghe, Bunker and Bhaskar, 2017). (Webster, 1958) was among the first to develop and apply the widely used concept today. (Koonce et al., 2008) describes SFR as the quotient of 3600 seconds period and the headway in seconds between successive vehicles departing from a queue as shown in (Eq. 3). In other words, saturation flow is as follows:

$S F R=3600 / h$

where $h$ is headway in seconds.

It typically begins in the middle portion of a green interval, specifically, 10 seconds after the interval turns green until the signal turns yellow or the queue clears (Akcelik, 1998; Ranasinghe, Bunker and Bhaskar, 2017). The importance of SFR to traffic operational analysis has attracted significant research efforts into the area. Since the pioneering work of (Greenshields, Schapiro and Ericksen, 1947), dozens of studies have been carried out in order to characterize saturation flow and understand its variability under different operating and geometric conditions, as well as various localities and communities.

SFR is primarily determined by two ways: estimates and field measurement. However, because data is often unavailable or prohibitively expensive to collect, SFR is typically determined using estimates. The Highway Capacity Manual (HCM) is the principal document used to arrive at these estimates. HCM assumes an ideal flow rate of 1,900 passenger cars/hour/lane. The factors that influence saturation flow can be categorized into traffic, geometric and area type variables. Based on the expected or observed presence of the above conditions, 
adjustment factors are then applied to the ideal value which either gives a downward adjustment or no effect on ideal flows. This is done in an attempt to properly reflect conditions other than the most ideal. The adoption of default ideal rates and adjustment factors for SFR calculations has several drawbacks primarily because they lead to erroneous results and inappropriate timing plans which can have severe consequences in cases where information is used for realtime traffic control and guidance systems (Shang, Zhang and Fan, 2014). (Turner and Harahap, 1993) noted that saturation flow rates in developing countries are noticeably different from those in developed countries largely because of four main factors: vehicle mix- variety of motorized and some nonmotorized vehicles with different operating characteristics, driver behaviour- poor lane discipline and adherence to traffic regulations (hence, undermining the first come first serve principle of many traffic models), public transport- a mix of buses of various sizes, loading and unloading locations and driving styles, and roadside activities- nearby land-uses which generate roadside parking and non-transport activities which ultimately reduces usable roadway space. Clearly, SFR has a highly localized nature and it is controlled largely by variables peculiar to each area's drivers, land use, vehicle type, traffic patterns and geometric layout. It is against this backdrop that many researchers have strongly recommended field measurement for SFR. (Webster and Cobbe, 1966) concluded that in order to reduce overall delay, SFR should be measured rather than estimated in order to reflect the impact of demographics, driver behaviour, and peculiar traffic conditions (Akcelik, 1998; Bonneson et al., 2005; Majeed et al., 2014). Since there is such a strong evidence base showing the variability of SFR in different spatial settings and operating conditions, it is imperative for transportation researchers, engineers and professionals to collect field data as much as possible to enable them to calibrate signal timing models and conduct performance analysis such as capacity, delay, throughput and queue length more accurately, reliably and confidently within their local environments. A plethora of studies have been carried out to determine the saturation flow rate. (Majeed et al., 2014) studied fifteen intersections in three cities in Alabama. They developed an empiricalbased exponential model to predict SFR and they found that number of through lanes, effective green time and volume per approach were the most influential factors in their model. The headways for the three cities were statistically different, even though they were in the same city. The mean headways for Huntsville, Birmingham and Montgomery were $2.477 \mathrm{~s}, 1.915 \mathrm{~s}$ and $2.786 \mathrm{~s}$ respectively. (Shao and Liu, 2012) studied 11 intersections in Beijing during the morning and evening peaks. Headway distribution in 8 sites followed a log-normal distribution while in 3 sites followed a normal distribution. They concluded that when headway data is asymmetrical, traditional headway methods yield underestimated SFR. They then developed a new method that incorporated the use of the median rather than the mean and this reflected the variance of headway around the estimate. (Arhin et al., 2016) studied SFR for various lane groups at 81 intersections in the District of Columbia. The average SFR for through, through and right, through and left and left movements were $1,559,1,461,1,566$ and $1,460 \mathrm{veh} / \mathrm{hr} /$ lane respectively. Similarly, a study of 36 signalized intersections in Beijing, China, (Shang, Zhang and Fan, 2014) found SFR to vary considerably by type of lane. SFR were $1,380 \mathrm{pc} / \mathrm{hr} /$ lane, $1,520 \mathrm{pc} / \mathrm{hr} /$ lane, 
$1,535 \mathrm{pc} / \mathrm{hr} /$ lane, $1,457 \mathrm{pc} / \mathrm{hr} /$ lane and $1,457 \mathrm{pc} / \mathrm{hr} /$ lane respectively for right turn, left turn, through lane, through-right and through-left lane respectively. Saturation headways in their work followed a normal distribution. This varied from the work of (Shao and Liu, 2012; Jin et al., 2009; Chodur, Ostrowski and Tracz, 2011) whose works showed headways to follow a log-normal distribution and (Alex and Isaac, 2014) whose work showed negative exponential headways in their study in India while (Zhao et al., 2015) have also established a logarithmic relationship between queue position and headway distribution. (Bara, Ahmad and Mohamad, 2021) studied SFR in Jordan and found SFR for shared Left and U-turn lanes to be $1,611 \mathrm{veh} / \mathrm{hr} /$ lane, through movement had SFR of $1,840 \mathrm{veh} /$ $\mathrm{hr} /$ lane while an overall average of 1,720 veh/hr/lane was observed. (Wang et al., 2020) studied SFR in Beijing, China and Washington, USA. The observed SFR varied quite significantly, across different operating conditions. SFR data was collected considering different intensities of heavy vehicles and lane widths. In this group of observations, the observed headway ranged between 1.99 - 4.6 seconds. SFR data was also collected considering the proportion of left-turning vehicles and lane widths. The observed headways for this group ranged between 1.5 - 5.5 seconds. In another recent study, (Kulakarni et al., 2020) estimated SFR at two intersections in Ahmedabad and Surat, India. SFR of $8842 \mathrm{veh} / \mathrm{hr}$ was calculated for a three-lane approach at one of the intersections using the TRL method. Clearly, different locations operating under a wide variety of conditions present varied sets of SFR and headways distributions.

Lagos is one of the most congested cities in the world owing to the presence of a relatively low road network/capita, high population, rapidly growing middle class, poor driver behaviour, and a huge demand for travel (Oni et al., 2008; Economic Intelligence Unit (EIU), 2013; LAMATA, 2021). Signalized intersections have generally been known to be critical points in the road network because they are more susceptible to congestion in comparison to other types of roads owing to its lower per lane capacity and the fact that the allotted green time is split amongst conflicting road users (Angel and Hickman, 2003). Additionally, signalized intersections accommodate many other types of transportation features such as bus stops, bicycle lanes and pedestrian crossings which may reduce capacity. SFR and headway are perhaps the most crucial parameters necessary to develop any reasonable understanding of the interaction between geometry, traffic operations, and signal timing. These aforementioned factors, alongside other factors such as weather, affect performance on roadways (Alhassan and Ben-Edigbe, 2011). A greater understanding of the characteristics of these parameters will increase the efficiency of allocation of green time to conflicting road users at signalized intersections. Therefore, making any congestion amelioration effort at signalized intersections in Lagos is more likely to succeed.

\subsection{Study Area}

Lagos is located between latitudes $6^{\circ} 22^{\prime} \mathrm{N}$ and $6^{\circ} 42^{\prime} \mathrm{N}$ and longitude $2^{\circ} 42^{\prime \prime} \mathrm{E}$ and $3^{\circ} 22^{\prime} \mathrm{E}$. It has a total area of $3,577.28 \mathrm{~km}^{2}$, a projected population of over 17 million, a population density of over 5,000 persons $/ \mathrm{km}^{2}$ and a growth rate of over $3 \%$ per annum (Filani, 2012; Lagos Bureau of Statistics, 2016). It is the most populous city in sub-Saharan African and one of the fastest-growing cities 
in the world. It is undoubtedly the economic nerve centre of Nigeria. As of 2006, Lagos accounted for $30 \%$ of Nigeria's GDP and $90 \%$ of foreign trade (Filani, 2012). The intense amount of economic activities performed in the state along with urbanization, a rapidly growing population, an expanding middle class, overall increase in income levels, and an endless supply of used vehicles have consequently given rise to increased demand for travel (EIU, 2013). Travel demand is estimated to be 20 million trips per day (LAMATA, 2021). About 99\% of these trips are carried out by road. The modal split of these road trips $20 \%$ private cars and taxis, over $70 \%$ public buses, and $9 \%$ motorcycles (LAMATA, 2021). Despite the huge population and demand for travel, the infrastructure to cater for this demand is still abysmally low, as the infrastructure stock is at a level that can serve just six million people. The state has a road network density of $0.4 \mathrm{~km} / 1000$. This is comparatively low even in Africa (LAMATA, 2021) and it is one of the major reasons for congestion, as 1.6 million vehicles ply Lagos roads daily (LASG, 2019). This results in a road density in Lagos of 226vehicles/ $\mathrm{km}$ in contrast to the national average of 16 vehicles/km (LASG, 2019; LAMATA, 2021).

The heavy vehicular traffic in Lagos has necessitated a significant presence of signalized intersections of varying sizes, geometric configurations and operating conditions. There is a total of One-hundred and thirty-six signalized intersections in Lagos (Lagos State Ministry of Transport, 2019). In this study, a total of eight approaches distributed across six intersections were selected in this study for analysis. Only the main approaches and straight movements were studied across all the selected approaches. Below is a brief description of the approaches and intersections and their locations are presented in Figure 1.

In summary, while Saturation Flow Rate (SFR) is unarguably a key traffic intersection performance assessment or prediction parameter, its estimation is subjective, being highly localized in nature as amply attested to in literature. In addition to determining appropriate values for Lagos traffic condition. The results obtained from this investigation have the potential for being preliminary estimates of SFR for many developing countries with similar traffic and intersection characteristics to that of Lagos, Nigeria. The results may also serve as bases for other specific investigations in such locations for which results are sparse or non-existent in literature.

Studies on SFR and headway in Lagos are very limited and for parameters such as these which are highly local, applying values imported from other locations incorrectly may result in adverse effects on operational performance. It will thus be of interest in this work to characterize headway and determine SFR in Lagos. This investigation seeks to address the open-ended research questions: What probability distribution(s) can best be utilized to characterize headways at signalized traffic intersections and hence determine saturation flow rates in Lagos, Nigeria? Is there a difference in saturation flow during peak and off-peak periods? To what extent do constraints such as pavement condition and bus stops affect saturation flow rates? The rest of this paper is organized as follows. Section 2 addresses the materials and methods adopted in this study. More specifically, section 2 covers the following: methods and procedures for data collection, equations used for headway and SFR calculation, normality 
test, tests of differences and fuzzy analysis of headway. Section 3 focuses on the Results. In the results section, the SFR and headway of different types of intersections studied, the results of normality tests, statistical test of differences, probability distributions, the effect of peak and off-peak periods on
SFR, the effect of traffic constraints on SFR and finally a simple fuzzy analysis of SFR are presented. Section 4 places the results produced in the context of similar literature and highlights limitations and future work. In section 5, conclusions are drawn on the subject matter.

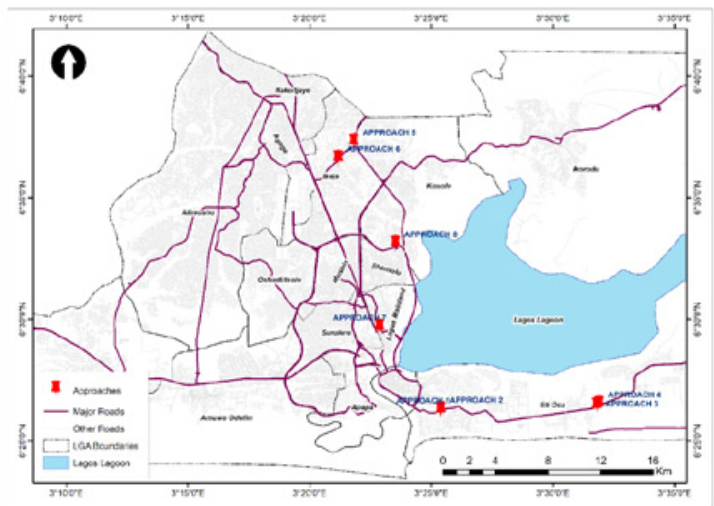

Fig. 1.

Location of Approaches Studied in Lagos Metropolis

\section{Materials and Methods}

\subsection{Data Collection}

Headway data were collected manually from six intersections and a total of eight approaches in Lagos Metropolis. The primary tool adopted for data collection was a digital Kadio (KD-6128) stopwatch accurate to $1 / 100^{\text {th }}$ of a second and a traffic counting sheet designed for the study. A snippet of the template adopted for the study is presented in Table 1.

The stopwatch was used to record the green times and the saturated portion of each cycle. The vehicles departing during the saturated period and the duration of the saturated period are then recorded in the template (Table 1) for extraction and further analysis. Microsoft Excel and XLSTAT 2018 software were adopted for data entry, cleaning and various tests of normality and statistical differences. Easy fit software was used for fitting headway observations into appropriate probability distributions.

Two categories of intersection approaches were selected for this study. First, ideal approaches, which are characterized by the following operating conditions:

- Limited or no pedestrian interference;

- No buses stopping or parking at the approach;

- Reasonably wide lanes of 3.5 - 3.6 metres;

- Good pavement condition; 
- A significant number of queued vehicles at the inception of the green interval (at least 15 during the peak periods);

- Limited or no turning interference affecting the through vehicles. If turning exists at the intersections, there are dedicated lanes present.

The SFR values obtained from ideal approaches could be considered the base SFR values upon which adjustment factors can be applied to obtain more realistic values that are due to traffic disturbances.

The second category is typical approaches, which represent approaches with disturbances such as buses and bus stops, pedestrian activities, hawkers, roadside parking, rough pavements and any other type of significant impediment to traffic flow. The typical intersections are more representative of traffic operations in Lagos metropolis since the ideal conditions are seldom observed. Four intersections were selected for the ideal group, and six approaches within these intersections were studied. Two intersections were selected from the typical group and two approaches within these intersections were studied. The two distinct groups were selected in order to provide a basis for comparing saturation flow rates.

Table 1

Saturation Flow Measurement Field Template

\begin{tabular}{|c|c|c|}
\hline Cycle Number & $\begin{array}{c}\text { Discharge from Queue during the Middle portion } \\
\text { of Green period }\end{array}$ & Saturation Time (secs) \\
\hline 1 & & \\
\hline 2 & & \\
\hline$\ldots$ & & \\
\hline
\end{tabular}

Saturation flow data were collected at various times throughout the day with interest in periods when the queues were long enough to provide a sustained discharge of vehicles during the green interval. However, efforts were made to collect data during periods of both light and heavy traffic. This was done to enable the study to identify the effect of peak period and time of day on SFR.

The headway data collection approach adopted was that recommended by (Akcelik, 1998). It is a simple method that involves counting the number of vehicles that are able to depart from a queue during a certain portion of the saturated green interval. The counting of vehicles that cross the stop line begins 10 seconds after the green interval for the approach of interest begins. The first ten seconds are excluded from the analysis in order to account for start-up lost time as recommended by the method. Cycles with the presence of trucks, or any abnormal event such as a vehicle suddenly stopping, a collision or vehicular breakdown were also excluded. Heavy vehicles were excluded because they often increase the headway of following vehicles and increase start-up lost times if they are in front of the queue because of their lower performance in start-up capabilities in comparison to smaller vehicles. Saturation time was taken as the amount of time required to clear the vehicles that were stopped during the previous red interval and including vehicles that arrive during the green interval but the vehicles in front of them is still stopped and the arriving vehicle is not more than one car length $(5 \mathrm{~m})$ 
from the leading vehicle. To achieve this, careful attention was given to note the last vehicle at the back of the queue during the red interval and the vehicles which join the queue during the green interval. In selecting which lane to analyse, at each chosen approach and intersection, the lanes with the least amount of interference such as lane changing or turning movements were selected. At some sites, this was the innermost lane among, while at others, it was the outermost lane. However, there was no observation of significant lane underutilization at any of the sites. Consequently, the results generated from the sample lanes are representative of the approaches of interest. Each selected lane was observed for a number of cycles during each hour (typically 15-30 cycles were observed each hour). In total, over 700 cycles were used for various analyses in this study.

\subsection{Headway and SFR Analysis}

SFR and headway analysis approach adopted is based on (Akcelik, 1998) and it is calculated as shown in (Equation 4):

$s f r=\frac{\sum s}{\sum t-\left(n_{c} \times 10\right)}$

where $s f r$ is saturation flow/second, $S$ is the number of vehicles departing during a saturated portion of the green, $t$ is the saturated green time and $n_{c}$ is the total number of cycles with valid observations.

When $s$ is calculated, it is then multiplied by 3600 to determine hourly SFR. Average headway was then calculated by rearranging (Eq. 3); since headway and SFR have an inverse relationship. Cycle by cycle headway was calculated using (Eq. 5):

$h_{i}=\frac{t_{i}-10}{S}$ where $h_{i}$ is headway in cycle $i, t_{i}$ is saturated green time for cycle $i$ and $S$ is the number of vehicles departing during a saturated portion of the green.

\subsection{Normality Test}

Shapiro-Wilks (hereafter S-W) test was performed in this study to check the normality of headway data. The W statistic is bounded by 0 and 1 . The closer the value is to 1 , the closer it is to the normal distribution and the closer it is to 0 , the closer it is to non-normal distribution.

\subsection{Test of Differences}

\subsubsection{Mann-Whitney U Test}

This is a non-parametric test used to determine if two samples are from the same population. It was adopted for analysis as a result of the non-normal and asymmetrical shape of headway data observed in this study.

If the $U$ statistic is $<\alpha$, then the alternative hypothesis that the groups are from statistically different populations or distributions is accepted. However, if the $\mathrm{U}$ statistic is $>\alpha$, then the null hypothesis is accepted. In this study, the $\alpha$ selected was 0.05 and a sample size of fifty-three (53) cycles was used for this test. This test was used to determine if headway from ideal and typical approaches were statistically different.

\subsubsection{Wilcoxon Signed-Rank Test}

In order to determine if a statistically significant difference exists between headway during the peak and off-peak for the same site, the Wilcoxon signed-rank test was adopted. This test is a non-parametric 
equivalent of a paired $t$-test which is used to test the difference between paired observations.

In this study, a sample size of One hundred and five (105) cycles was adopted for this test.

\subsection{Fuzzy Analysis of Headway}

Level 1 fuzzy analysis (6) was performed on headway data at ideal approaches to establish a defuzzified headway for each approach. A total of 360 cycles from a total of four different intersections was used for the analysis. The defuzzified headway, $d h$ is given by (Eq. 6):

$d h=C-[0.5 \times((C-A) \times(C-B))]^{1 / 2}$

where $C$ is the maximum of headway observations, $A$ is the minimum of observations and $B$ is the mean.

\section{Results}

\subsection{Analyses of Typical and Ideal Approaches}

As shown in Table 2, approaches with ideal operating conditions typically have the highest saturation flow rates which are comparable to and even higher than observations in more developed countries. A preliminary field survey in Lagos has shown that such approaches with ideal or near-ideal conditions are very few in Lagos and they represent less than $10 \%$ of the total number of intersections present in the state.
For example, the difference in saturation flow rates at approaches 6 and 7 was 461 $\mathrm{veh} / \mathrm{hr} /$ lane while the difference between them at approaches 6 and 8 was 750 veh/ $\mathrm{hr} /$ lane. Such a large difference in SFR is sufficient to warrant an additional lane of travel. The presence of a bus-stop and poor pavement conditions at a typical approach, particularly approach 8 , appeared to have influenced the largest difference in SFR between ideal and typical approaches in this study. Bus stops located downstream of the stop-line on typical approaches typically reduce the number of discharge lanes downstream by one since buses park on a lane to allow passengers to board or alight. Figure 2 presents the comparison between headways on an Ideal and a Typical Intersection approach.

Mann-Whitney two-tailed test was performed to determine whether there was a statistically significant difference in headway at ideal and typical approaches. A p-value $<0.0001$ was obtained from the analysis at an alpha level of 0.05 . This further provides evidence to support the claim that headways at ideal and typical approaches vary markedly.

Evidently, there are substantial variations between the headways on the approaches of the two groups with the Typical having higher headway values over almost all the observation cycles. Table 2 shows the Saturation flows, headways, standard deviation of headway and other characteristics of the investigation over several cycles. 


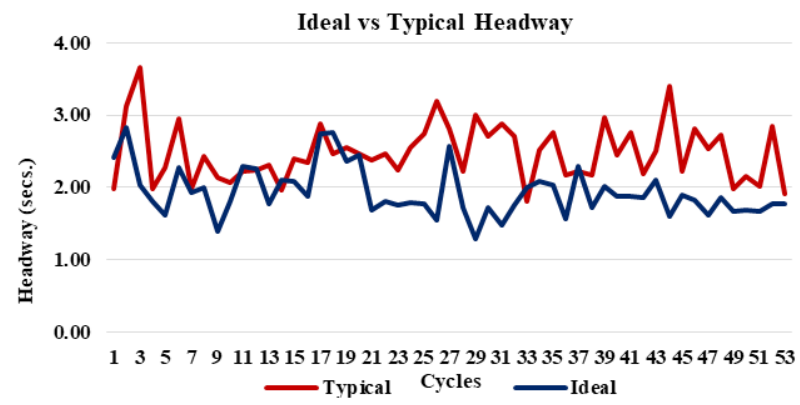

Fig. 2.

Comparison between Headways at an Ideal Approach and a Typical Approach

Table 2

Saturation Flow, Headway and Intersection Characteristics

\begin{tabular}{|c|c|c|c|c|c|c|}
\hline ApproachCode & $\begin{array}{c}\text { Saturation Flow } \\
(\mathbf{v e h} / \mathbf{h r} / \mathbf{l a n e})\end{array}$ & $\begin{array}{c}\text { Headway } \\
(\mathbf{h})(\mathbf{s e c s})\end{array}$ & $\begin{array}{c}\text { Standard } \\
\text { Deviation of } \\
\text { Headway }\end{array}$ & $\begin{array}{c}\text { Intersection } \\
\text { Condition }\end{array}$ & $\begin{array}{c}\text { Traffic } \\
\text { Condition }\end{array}$ & $\begin{array}{c}\text { Sample } \\
\text { Size (Cycles) }\end{array}$ \\
\hline 1 & 1,629 & 2.21 & 0.45 & Ideal & Off-peak & 72 \\
\hline 2 & 1,921 & 1.87 & 0.38 & Ideal & Peak & 87 \\
\hline 3 & 1,935 & 1.86 & 0.31 & Ideal & Peak & 154 \\
\hline 4 & 1,746 & 2.06 & 0.34 & Ideal & Off-peak & 57 \\
\hline 5A & 1,906 & 1.89 & 0.43 & Ideal & Peak & 107 \\
\hline SB & 1,669 & 2.16 & 0.7 & Ideal & Off-peak & 109 \\
\hline 6 & 1,947 & 1.85 & 0.19 & Ideal & Peak & 22 \\
\hline 7 & 1,486 & 2.42 & 0.4 & Typical & Peak & 53 \\
\hline 8 & 1,197 & 3.00 & 0.64 & Typical & Peak & 45 \\
\hline
\end{tabular}

\subsection{Normality, Probability Distributions and Headway Characteristics}

Normality tests were performed on cycle to cycle average headway using the SW test. The p-values for the various approaches are as follows: Approaches $1(<0.0001)$, 2 (<0.00010), 3 (<0.0001), 4 (0.948), 5A $(<0.0001), 5 \mathrm{~B}(<0.0001), 6(0.055), 7$ (0.121) and $8(0.016)$. The $\mathrm{p}$-values show that headways on only three approaches follow a normal distribution while those on the remaining approaches follow non-normal distributions. However, it may be possible that the deviation from the normality of headways on these three sites was only small to moderate and the sample sizes were not large enough to detect any non-normality. As noted by (NCSS Statistical Software, 2020) large sample sizes may be necessary to detect small to moderate deviations from normality. Headways on approaches 4, 6, and 7 which follow normal distribution based on SW had sample sizes of 56,22 , and 53 respectively. Therefore, it is a possibility that the relatively smaller sample sizes of these sites contributed to the acceptance of normality by the SW test. Nevertheless, it became necessary to adopt a more general distribution that is capable of reflecting the variation in headway under different prevailing conditions. 
The normal distribution was not a good fit for the headway data collected at five of the approaches surveyed. The results of the analysis performed showed that cycle to cycle headway data is asymmetrical, as the mean and median were essentially different. Furthermore, all headway distributions showed positive skew of between 0.67 and 1.44. This further strengthens the argument that headway data is non-normal. The only approach which deviated from the pattern was approach 4 with a skew of -0.005 . This approach had a higher median headway compared to the mean, although, the difference was relatively small.

The lognormal model was selected to model the headway cycle to cycle average headways. It was capable of modelling all approach data at alpha levels 0.05 except for intersection 1. Surprisingly, the Burr group of distributions were found to model headway at all the approaches more accurately and during different periods quite well with a significantly better fit than all the other common probability models for headway such as lognormal, normal and negative exponential model. Figure 3 shows Burr fit for data at approach 6. The long tails towards the right and the bunching of most data to the left is what makes Burr fit a good approximation for headway data. For a Burr distribution, the Probability distribution function (pdf) and Cumulative distribution function (cdf) of any value of $x$ is obtained by (Eq. 7 and 8) respectively.

$f(x)=\frac{\alpha \times k\left(\frac{x}{\beta}\right)^{\alpha-1}}{\beta\left(1+\left(\frac{x}{\beta}\right)^{\alpha}\right)^{k+1}}$

$f(x)=1-\left(1+\left(\frac{x}{\beta}\right)^{\alpha}\right)^{-k}$

where $k$ and $\alpha$ are location parameters and $\beta$ is a scale parameter.

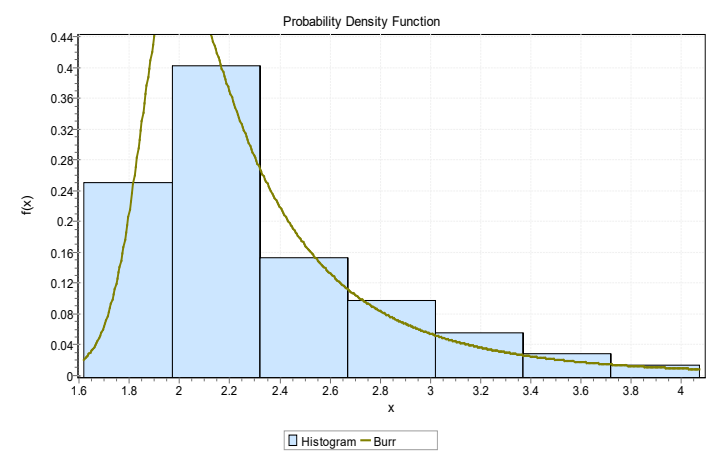

Fig. 3.

Cycle to Cycle Headway (Obafemi Awolowo)

\subsection{The Effect of Peak and Off-peak Period on Saturation Flow Rates and Headway}

Traffic condition was observed to have a strong effect on saturation flow rate. During periods of higher flows and longer queues (peak period), headways were smaller and hence saturation flow rates were significantly higher (Figure 4). The analysis performed on approach 5 during the peak and off-peak periods showed very striking differences during the two periods. 


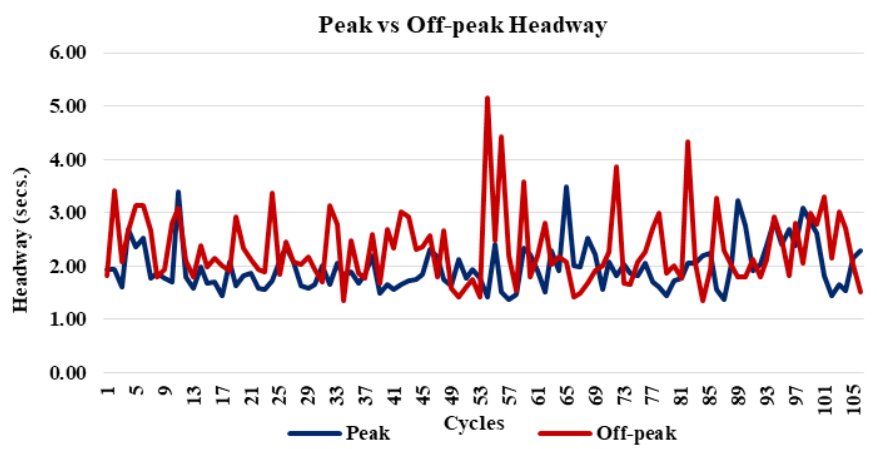

Fig. 4.

Comparison of AM (Peak) and PM (Off-peak) Average Headway/Cycle

The difference in saturation flow was as high as $237 \mathrm{veh} / \mathrm{hr} /$ lane. Normality tests on headway data at approach 5 from both morning and afternoon sessions showed they were non-normal.

The results of the Wilcoxon signed-rank test showed that morning and afternoon headway data are not from the same population with a p-value $<0.0001$ at an alpha level of 0.05 . In other words, headway for morning and afternoon did not vary by chance.

Similarly, the Mann-Whitney test was applied to two independent approaches (1 and 2). The resulting p-value was $<0.0001$ at an alpha level of 0.05 . This further provides supporting evidence to conclude that headways vary during peak and offpeak periods.

\subsection{Fuzzy Analysis of Headway}

The fuzzy analysis performed on the measured headways at ideal approaches generally resulted in a headway increase of $1-15.8 \%$ and an SFR reduction of $1-13.75 \%$ (Table 3). Therefore, giving rise to a more conservative SFR estimate. Additionally, fuzzy analysis showed significant deviation from the mean. The ideal approaches with larger standard deviations and higher maximum headway values (approaches 2 and 5) had significantly more conservative defuzzified headways in comparison to approaches 3 and 6, even though the mean values were all very close. This had a significant impact on the resulting SFR. SFR at approaches 2 and 5 resulted in a difference of $262 \mathrm{veh} / \mathrm{hr} / \mathrm{ln}$ and $199 \mathrm{veh} / \mathrm{hr} /$ lane respectively when the fuzzy and mean based SFR were compared.

Table 3

Defuzzified Headway (dh) and SFR for İdeal Approaches

\begin{tabular}{|c|c|c|c|c|c|c|c|}
\hline s/n & $\begin{array}{c}\text { Approach Name } \\
\text { \& Code }\end{array}$ & $\begin{array}{c}\text { Mean } \\
(\mathbf{d h})\end{array}$ & $\begin{array}{c}\text { Defuzzified } \\
(\mathbf{d h})\end{array}$ & $\begin{array}{c}\mathbf{\%} \\
\mathbf{D i f f}\end{array}$ & $\begin{array}{c}\text { Mean based } \\
\text { SFR }\end{array}$ & $\begin{array}{c}\text { Defuzzified } \\
\text { SFR }\end{array}$ & $\begin{array}{c}\text { \% } \\
\text { Diff }\end{array}$ \\
\hline 1 & Lekki to Ajah - 3 & 1.86 & 1.88 & 1.07 & 1,935 & 1,915 & 1.03 \\
\hline 2 & MKO - 5 & 1.89 & 2.19 & 15.87 & 1,906 & 1,644 & 13.75 \\
\hline 3 & Obafemi Awolowo - 6 & 1.85 & 1.92 & 3.78 & 1,947 & 1,875 & 3.7 \\
\hline 4 & Ozumba to bonny - 2 & 1.87 & 2.09 & 11.7 & 1,921 & 1,722 & 10.36 \\
\hline
\end{tabular}




\section{Discussions}

The results of this study have important practical and theoretical implications which are highlighted subsequently. Headway data were non-normal and this suggests that the mean may not be the best parameter for estimating headway for such observations, as it may be affected by a few outliers (Sedgwick, 2010; Manikandan, 2011). Therefore, care needs to be taken in applying the mean for headway modelling. The median may typically be more representative in such cases since about half of the observations will be lower than the mean. (Shao and Liu, 2012) also highlighted a similar finding in their work. When a mean or median headway value was adopted to calculate SFR, the Base SFR and headway in this study (> $1900 \mathrm{veh}$ / $\mathrm{hr} / \mathrm{ln}$ ) were comparable to or higher than SFR in developed countries. However, headway is associated with a certain level of uncertainty particularly because in addition to many of the aforementioned factors, it is also dependent on driver behaviour. Driver behaviour has been shown to vary by age and sex as well as other more abstract factors such as personality traits, experience and even the driver's state of mind (Willemsen et al., 2008), all of which are difficult, if not impossible to quantify in the field. As a result of the uncertainties associated with headway and invariably SFR, adopting the mean or median may lead to erroneous results and suboptimal performance at intersections. Consequently, a fuzzy approach was also adopted. This yielded a much lower value than the mean SFR. The average SFR observed for four ideal approaches considering a fuzzy approach was $1,789 \mathrm{veh} / \mathrm{hr} / \mathrm{ln}$ while adopting a mean based approach yielded $1,927 \mathrm{veh} / \mathrm{hr} / \mathrm{ln}$. The fuzzy approach should be considered superior since it captures the various uncertainties that may be difficult to explain or measure. The analysis showed that the mean and fuzzybased SFR estimates vary considerably. Clearly, such a large difference in SFR will have a significant effect if one is adopted instead of the other.

Burr distribution provided the best fit for headway distribution. The high performance of the Burr distribution is in keeping with the findings of (Alhassan and Ben-Edigbe, 2011) who also showed that Burr was the best distribution for both rainy and non-rainy conditions in their study. Burr distribution has been adopted quite well in travel time studies, reliability engineering, finance, hydrology and insurance. It was well suited to headway data because of the presence of long tails towards either the left or the right of observations. This is because as headway values begins to increase or decrease and as the likelihood of observing higher or lower headway reduces, the long tails at either end may become more pronounced.

The time of day also has a noticeable effect on headway. The significant variation between headway at different times of the day is supported by (Bonneson et al., 2005) which suggests that drivers typically behave differently during peak and off-peak periods. This variation was probably due to the aggressiveness of drivers in Lagos during the peak period. In order to arrive at work early during the early rush hours, drivers may follow leading vehicles more aggressively, therefore reducing headway. Additionally, there was a larger variation in headways during the off-peak in comparison to the peak. The largest standard deviations were 0.7 seconds and 0.5 seconds respectively, and they were both observed during the off-peak (Table 2). The fact that headway and invariably SFR vary with time of day suggests that modelling efforts should reflect 
this because indeed, SFR is assumed fixed in most modelling and simulation efforts and this could produce erroneous outcomes.

The SFR reduction effects of buses and pavements observed in this study are not particularly surprising because after all, bus stops have been shown to reduce the SFR (Ranasinghe, Bunker and Bhaskar, 2017) and capacity at signalized intersections (Ghasemlou et al., 2012; Ghasemlou, Aydin and Yildrim, 2016) quite noticeably. The pulling-out and pulling-in of buses also cause additional friction for straight movement, as they constantly need to decelerate to permit pull-out and pull-in. The presence of bus stops resulted in a noticeable difference of $\leq 460 \mathrm{veh} / \mathrm{hr} / \mathrm{ln}$ between an ideal and a typical approach in this study. However, based on the results, poor pavement appears to have a far more significant impact on SFR, as $\mathrm{SFR}$ reduced by $\leq 750 \mathrm{veh} / \mathrm{hr} / \mathrm{ln}$ when compared to the ideal approach. (Ben-Edigbe and Ferguson, 2005, 2009; Ghasemlou, Aydin and Yildrim, 2016; Aydin and Topal, 2019), have shown that poor pavement has a significant effect on capacity and traffic flow conditions at signalized intersections. Therefore, the significantly reduced SFR observed due to poor pavement is supported by findings in other studies.

The results of this study are of significant importance to timing plan development, since it provides a basis for optimal green time calculation, through the selection of the most appropriate SFR and headway for a variety of operating conditions. Clearly, adopting a saturation flow rate without field checks even within the same city will lead to erroneous results with severe consequences on the operational performance of an intersection.
The few sites studied and the lack of data on several other factors which affect SFR is a limitation of this study. This limits the ability to establish quantitative relationships between SFR and constraining variables. Further work which will cover a larger number of intersections is necessary to gather sufficient data for robust model development is encouraged. A new model or a modification/extension of the current HCM saturation flow model should be developed for Lagos and perhaps other developing countries to enable the accurate quantification of the effects of traffic-flow constraining variables in their peculiar environments. These new models should also seek to identify the principal factors affecting saturation flow such that they are concise and data inputs are as few as possible to minimize data collection requirements. In addition, the spatial transferability of saturation flow models should be explored as this might be quite useful in locations where operating conditions are similar and resources might be limited to embark on comprehensive studies for new model development.

\section{Conclusion}

This study characterized headway and saturation flow rates at eight signalized intersection approaches and over 700 signal cycles in Lagos Metropolis, Nigeria. The data were subjected to statistical tests and probability fitting. Based on the results of this study and previous literature, we conclude that the Burr group of distributions although seldom used in headway modelling, is a suitable distribution due to its ability to accurately model phenomena with long tails. It should therefore be considered as an alternative to other common probability distributions for headway modelling in urban cities with similar 
traffic characteristics with Lagos, Nigeria. The base SFR varied considerably between the mean-based and fuzzy-based approach and headway data was generally skewed. Therefore, the mean may not be the optimal statistic to represent SFR and headway.

Headway and SFR vary significantly spatiotemporally, and this should be reflected during timing plan development and simulation or modelling exercises. The findings of this study are key and important inputs for design, optimization and performance analysis at signalized intersections. Traffic engineers may see a significant uptick in traffic operations performance from simply adopting the appropriate headway and SFR values which truly reflect operating conditions.

\section{References}

Akcelik, R. 1998. Traffic Signals: Capacity and Timing Analysis. 123. Vermont South, Victoria, Australia: Australian Roads Research Board (ARRB), Seventh reprint, 108p.

Agent, K.R.; Crabtree, J.D. 1982. Analysis of saturation flow at signalized intersections. UKTRP-82-8. Frankfort: Kentucky Transportation Center, 43p.

Angel, A.; Hickman, M. 2003. A Method for Analyzing the Performance of Signalized Intersection from Airborne Imagery', in. Transportation Research Board 82nd Annual Meeting, Washington, D.C. 20p.

Alex, S.; Isaac, K.P. 2014. Traffic simulation model and its application for estimating saturation flow at signalized intersection, International Journal for Traffic and Transport Engineering, 4(3): 320-338. doi:http:// dx.doi.org/10.7708/ijtte.2014.4(3).06.

Alhassan, H.M.; Ben-Edigbe, J. 2011. Effect of Rain On Probability Distributions Fitted to Vehicle Time
Headways, International Journal on Advanced Science Engineering and Information Technology, 2(2): 31-37. doi:10.18517/ijaseit.2.2.173.

Arhin, S. et al. 2016. Prevailing saturation flow rates for lane groups in urban areas, International Journal for Traffic and Transport Engineering, 6(2): 231-242. doi:http:// dx.doi.org/10.7708/ijtte.2016.6(2).10.

Aydin, M.M.; Topal, A. 2019. Effects Of Pavement Surface Deformations On Traffic Flow, Transport, 34(2): 204-214. doi:https://doi.org/10.3846/ transport.2019.8631.

Bara, W.A.; Ahmad, H.A.; Mohamad, S.A. 2021. Investigation of saturation flow rate using video camera at signalized intersections in Jordan, De Gruyter Open Eng, 11(1):216-226. doi:https://doi.org/10.1515/eng2021-0021.

Ben-Edigbe, J.; Ferguson, N. 2005. Extent of capacity loss resulting from pavement distress, Proceedings of the Institution of Civil Engineers - Transport, 158(1): 27-32. doi:https://doi.org/10.1680/tran.2005.158.1.27.

Ben-Edigbe, J.E.; Ferguson, N.S. 2009. Qualitative road service reduction resulting from pavement distress, in. WIT International Conference on Urban Transport, Bologna.

Bonneson, J. et al. 2005. Guidelines for quantifying the influence of area type and other factors on saturation flow rates. Research Report PR9385-V2. Tallahassee, USA: Florida Department of Transport, $88 \mathrm{p}$.

Chodur, J.; Ostrowski, J.; Tracz, M. 2011. Impact of saturation flow changes on performance of traffic lanes at signalized intersections, Procedia Social and Behavioural Sciences, 16(6); 600-611. doi:https://doi.org/10.1016/j. sbspro.2011.04.480.

Economic Intelligence Unit (EIU). 2013. The Socioeconomic Costs of Traffic Congestion in Lagos. Working Paper Series No 2. Lagos: Ministry of Economic 
Planning \& Budget, Lagos State, 14p. Available at: http://mepb.lagosstate.gov.ng/wp-content/uploads/ sites/29/2017/01/EIU-Working-Paper-2-Draft-2Traffic-Congestion.pdf.

Filani, M.O. 2012. The Changing Face of Lagos: From Vision to Reform and Transformation. Brussels, Belgium: Cities Alliance: Cities Without Slums, 44p. Available at: https://www.citiesalliance.org/sites/ default/files/Lagos-reform-report-lowres.pdf(Accessed: 18 February 2021).

Fornalchyk, Y.; Mohyhla, I.; Hilevych, V. 2013. Saturation flow volume as a function of intersection passing speed, Transport problems, 8(3): 43-52.

Ghasemlou, K. et al. 2012. Effect of Dwell Time on Performance of Signalized Intersections, in. 10th International Congress on Advances in Civil Engineering, Ankara, Turkey. 1-10.

Ghasemlou, K.; Aydin, M.M.; Yildrim, M.S. 2016. An Investigation of Lane Blockage Effects at Signalized Intersections, International Journal for Traffic and Transport Engineering, 6(3): 289-302. doi:10.7708/ ijtte.2016.6(3).05.

Greenshields, B.D.; Schapiro, D.; Ericksen, E.L. 1947 Traffic Performance at Urban Street Intersections. Technical Report 1. Yale Bureau of Highway Traffic, Eno Foundation for Highway Traffic Control. 23-30.

Highway Capacity Manual (2010). Fifth edition. Washington, D.C: Transportation Research Board.

Jin, X. et al. 2009. Departure headways at signalized intersections: A log-normal distribution model approach, Transportation Research Part C: emerging technologies, 17:318-327. doi:10.1016/j.trc.2009.01.003.

Koonce, P. et al. 2008. Traffic Signal Timing Manual. FHWA-HOP-08-024. U.S. Department of Transportation- Federal Highway Administration, 265p. Available at: https://nacto.org/docs/usdg/signal_ timing_manual_fhwa.pdf (Accessed: 19 April 2016).

Kulakarni, R. et al. 2020. Estimation of Saturation Flow at Signalized Intersections Under Heterogeneous Traffic Conditions, In Transportation Research, Lecture Notes in Civil Engineering, 45: 591-605. doi:https://doi. ord/10.1007/978-981-32-9042-6_47.

Lagos Bureau of Statistics (2016) Abstract of Local Government Statistics. Lagos State: Ministry of Economic Planning and Budget, 94p.

Lagos State Ministry of Transport (2019) Signalized Intersections in Lagos State. Unpublished Internal Report.

LAMATA. 2021. Available at: https://lamata.lagosstate. gov.ng/ (Accessed: 18 February 2021).

LASG (2019). Available at: https://lagosstate.gov.ng/ blog/2019/08/29/transport-commissioner-says-over1-6m-vehicles-ply-lagos- roads-daily/ (Accessed: 18 February 2021).

Majeed, A.A. et al. 2014. Field-based saturation headway model for planning level applications, International Journal of Traffic and Transportation Engineering, 3(5): 207-215. doi:10.5923/j.ijtte.20140305.01.

Manikandan, S. 2011. Measures of central tendency: Median and mode, J Pharmacol Pharmacother, 2(3): 214-215. doi:10.4103/0976-500X.83300.

NCSS Statistical Software. 2020. 'Normality Tests'. Available at: https://pdf4pro.com/view/chapter-194normality-tests-ncss-5b7449.html (Accessed: 12 May 2020).

Oni, S.I. et al. 2008. A Daily Flow Profile of Traffic in an Urban Traffic Corridor: The Nigerian Experience, Indus Journal of Management \& Social Sciences, 2(2):99-109.

Ranasinghe, W.; Bunker, V.; Bhaskar, A. 2017. Saturation headway variation at a signalized intersection approaches 
with a downstream bus stop and bicycle lane, in. Australian Transport Research Forum Proceedings, Auckland.

Sedgwick, P. 2010. Skewed distributions, BMJ [Preprint], (341). doi:https://doi.org/10.1136/bmj.c6276.

Shang, H.; Zhang, Y.; Fan, L. 2014. Heterogeneous lanes' saturation flow rates at signalized intersections, Procedia Social and Behavioural Sciences, 138, pp. 3-10. doi:10.1016/j.sbspro.2014.07.175.

Shao, C.; Liu, X. 2012. Estimation of saturation flow rates at signalized intersections, Discrete Dynamics in Nature and Society, 9 p. doi:https://doi. org/10.1155/2012/720474.

Stokes, R., W. 1988. Comparison of Saturation flow rates at signalized intersections, ITE journal, Institute of Transportation Engineers, 58(11):15-20.

Turner, J.; Harahap, G. 1993. Simplified saturation flow data collection methods. Research Report PA1292/93. Crowthorne: Transport Research Laboratory, $12 \mathrm{p}$.
Wang, Y. et al. 2020. An Analysis of the Interactions between Adjustment Factors of Saturation Flow Rates at Signalized Intersections, Sustainability, 12(2): 665. doi: $10.3390 /$ su12020665.

Webster, F.V. 1958. Traffic Signal Settings. Road Research Technical Report No.39. Her Majesty's Stationery Office, London, England: Road Research Laboratory, $45 \mathrm{p}$.

Webster, F.V.; Cobbe, B.M. 1966. Traffic Signals. Road Research Technical Report No. 56. Her Majesty's Stationery Office, London, England: Road Research Laboratory, 111p.

Willemsen, J. et al. 2008. The Dula Dangerous Driving Index: An Investigation of Reliability and Validity across Cultures, Accid Anal Prev, 40(2): 798-806. doi:10.1016/j. aap.2007.09.019.

Zhao, Y. et al. 2015. Estimation of saturation flow rate and start-up lost time for signal timing based on headway distribution, Discrete Dynamics in Nature and Society, $7 \mathrm{p}$. doi:https://doi.org/10.1155/2015/304823. 